\title{
Precision Analysis of an Analytical Method in Space Debris Orbit Prediction
}

\author{
Bin Wang ${ }^{1}$, a, Huafeng Peng ${ }^{1}$ and Kun $\mathrm{Li}^{2}$ \\ ${ }^{1}$ National Key Laboratory of Science and Technology on Blind Signal Processing, Chengdu \\ 610041, China; \\ ${ }^{2}$ Southwest Electronics and Telecommunication Technology Research Institute, Chengdu 610041, \\ China. \\ awbin93@163.com
}

\begin{abstract}
Using high precision laser ephemeris as reference, the precision of orbit prediction is analyzed of an analytical model which is formulated by quasi-analytical average method. The result shows that: the orbit prediction accuracy of MEO and LEO satellites is several hundred meters in 1 day and not more than $10 \mathrm{~km}$ in 7 days. The position error changes quickly over time. The calculation speed is fast using the analytical model. It can be used for precise orbit determination for a short term about 1 or 2 days. And it also can be used for the general accuracy of the long-term orbit prediction. But if the prediction time is too long, the accuracy will drop sharply.
\end{abstract}

Keywords: Spacecraft; Orbit prediction; Precision analysis; Quasi-analytical average method.

\section{Introduction}

The space environment has been more and more complex as the frequent space activities of the human beings. The vast majority of space objects are space debris except a little part of working spacecraft. At present, the numbers of space junk which can be detected are nearly 17000 . These huge amounts of space objects have caused a great threat to the operation of the spacecraft. Therefore, it is necessary to track and forecast the orbits of space debris.

Common orbit determination and prediction methods are numerical and analytical methods, the numerical method is of high accuracy, but the operation speed is slow. Considering timeliness requirement of orbit calculation of some application environment, the analysis method of calculation can be chosen which speed is faster. The SGP4 and SDP4 models were published by the North American Air Defense Command (NORAD) with refinements over the original model to handle the larger number of objects in orbit. These models predict the effect of perturbations caused by the Earth's shape, drag, radiation, and gravitation effects from other bodies such as the sun and moon. They have been widely used for a long time, but should be used with the two-line element. So the application is limited. The accuracy and reliability of the prediction of the SGP4/SDP4 model were studied and analyzed in detail in the literature [1 2]. In this paper, another analytical model which is formulated by quasi-analytical average method is studied. With the similar research methods mentioned in the literature, the prediction accuracy of the proposed model is analyzed in detail, which provides a reference for the relevant application research.

\section{Orbit Prediction Model}

In addition to orbit maneuver, satellites in the space are subjected to some kind of natural forces. The main of the forces is the earth's gravity. The other kinds of forces can be attributed to perturbation. If we can get the exact model of the different kinds of perturbation force, the state of motion of a satellite at any given moment can be obtained. But in fact, the expressions of various forces are very complex. At present, in addition to the two body movement, there is no strict analytical solution of any other kinds of perturbation. Therefore, under the current conditions, the numerical method is used to solve the equation of state of the satellite. But the analysis method has the important significance of its research on the advantage of the computation speed and the expression of the change law of the orbit movement. And then, the quasi-analytical average method is introduced. 


\subsection{Quasi-analytical Average Method.}

The quasi-analytical average method is an improvement of the average method. And it has solved the singularity problem. The difference between these two methods is the selection of the reference solution. The definition and form of the quasi average root number are as follows:

$$
\bar{\sigma}(t)=\bar{\sigma}^{(0)}(t)+\sigma_{c}(t)+\Delta \sigma_{l}^{(1)}(t)+\cdots
$$

The eccentricity ratio of the earth orbit satellite is often small, that is, the perturbation analysis of the Kepler's root number in the near circle orbit is often faced with the problem of the geometric singularity. In order to eliminate the singularity caused by the small eccentricity, the first kind of nonsingular variable is used in the paper to adapt to any eccentricity [4]. The expression form of the first kind of non- singular variable is as follows:

$a, i, \Omega, \xi=e \cos \omega, \eta=e \sin \omega, \lambda=M+\omega$

\subsection{Perturbation Force Model}

In order to achieve certain prediction accuracy, the orbit calculation must take into account the perturbation term. The accuracy of the model depends upon the perturbation solution of each source generated by perturbation. The higher the accuracy of the model is, the more complex the perturbation solution is. The analytical method used in this model mainly considers perturbation terms as follows:

1) The non-spherical gravity.

2) Lunar and solar perturbations.

3) Atmospheric drag perturbation

Each main perturbation source is corresponding to a set of perturbation analysis solutions. The time system used in this paper is UTC and the coordinate system is J2000.

\section{Ephemeris References}

The ephemeris reference used in this paper is precise ephemeris data of CPF format downloaded from the NASA website [5]. Seven days of five LEO and MEO satellites' orbit data are chose to be the reference resources. The CPF format ephemeris of ILRS is adopted in earth fixed coordinate system. The ephemeris data is transformed to J2000 geocentric celestial coordinate system and then compared with the ephemeris predicted by the analytical method. The basic information of the selected reference satellite is shown below in Table 1.

The first set of position and velocity data of the precise ephemeris is converted to the classic orbit elements as the initial value of the calculation of ephemeris by analytical method. The prediction interval is adjusted according to the reference satellite ephemeris. The output is position and velocity data of seven days of the satellite. Temporal inconsistency is handled by interpolation during the comparison with the precise ephemeris.

Table1 Satellites with precise ephemeris data used in our experiments

\begin{tabular}{cccccc}
\hline $\begin{array}{c}\text { Satellite } \\
\text { Name }\end{array}$ & NORAD Number & Altitude/km & Eccentricity & Inclination/deg & Data start date \\
\hline Larets & 27944 & 691 & 0.0002 & 98.204 & $2005-11-29$ \\
Ers2 & 23560 & 800 & 0.001 & 99.0 & $2005-11-29$ \\
Ajisai & 16908 & 1485 & 0.001 & 50.0 & $2005-11-29$ \\
Lageos1 & 8820 & 5850 & 0.0045 & 110.0 & $2005-11-29$ \\
Etalon1 & 31115 & 19105 & 0.00061 & 65.0 & $2005-11-29$ \\
\hline
\end{tabular}

\section{Precision Analysis of Prediction}

In this part, 1 day forecast RMS of the position difference and 7days forecast error curve are analyzed. Some valuable conclusions are drawn after the comparison between the satellites with different heights.

\subsection{Short-time Prediction}

First, ephemeris of prediction by analytical and the reference data were transformed into the same coordinate system (J2000). And then, statistical analysis shows the RMS of the position difference 
from $\mathrm{X}, \mathrm{Y}$ and $\mathrm{Z}$ direction. If the ephemeris data length is $k$, the position deviation between the ephemeris calculated by analytical and standard ephemeris is $y_{i}$.Then the RMS of the position difference is as bellow.

$$
R M S=\sqrt{\sum_{i=1}^{k} y_{i}^{T} W_{i} y_{i} / k}
$$

The statistical results with 1days of ephemeris error are shown in Table 2 below.

Table2 Position difference of prediction in 1 day

\begin{tabular}{ccccccc}
\hline Satellite Name & & Larets & Ers2 & Ajisai & Lageos1 & Etalon1 \\
\hline Altitude/km & & 691 & 800 & 1485 & 5850 & 19105 \\
& $\mathrm{X}$ & 382 & 363 & 618 & 212 & 786 \\
\cline { 2 - 6 } Position difference/m & $\mathrm{Y}$ & 363 & 367 & 345 & 262 & 514 \\
& $\mathrm{Z}$ & 164 & 286 & 476 & 266 & 1034 \\
& Total & 552 & 590 & 853 & 430 & 1397 \\
\hline
\end{tabular}

We can draw the following conclusions from table 2.

1) The RMS of position error for the MEO and LEO satellites of 1 day prediction using the analytical method is not more than $1 \mathrm{~km}$. That means this model has a good short-term forecasting accuracy.

2) In the forecast period of one day, the overall prediction accuracy is about several hundred meter scale. This analytical model can be used for precision orbit determination of general high precision. But the arc length of the observation data should not be more than one day.

3) The area to mass ratio of the satellite was not corrected during the prediction. If this parameter can be estimated during the precise orbit determination, the accuracy of ephemeris will be better.

\subsection{Long-time Prediction}

In order to analyze the accuracy of the model for long term prediction, the ephemeris was predicted for seven days using the initial orbit element and then compared with the reference ephemeris. The seven days position error curve is shown in the Fig1-5. Meanwhile, the formula for calculating the position error is as follows.

$$
\Delta l=\sqrt{\left(x-x_{0}\right)^{2}+\left(y-y_{0}\right)^{2}+\left(z-z_{0}\right)^{2}}
$$

Here, $(x, y, z) 、\left(x_{0}, y_{0}, z_{0}\right)$ were the prediction position by analytical method and the reference position of the satellites.

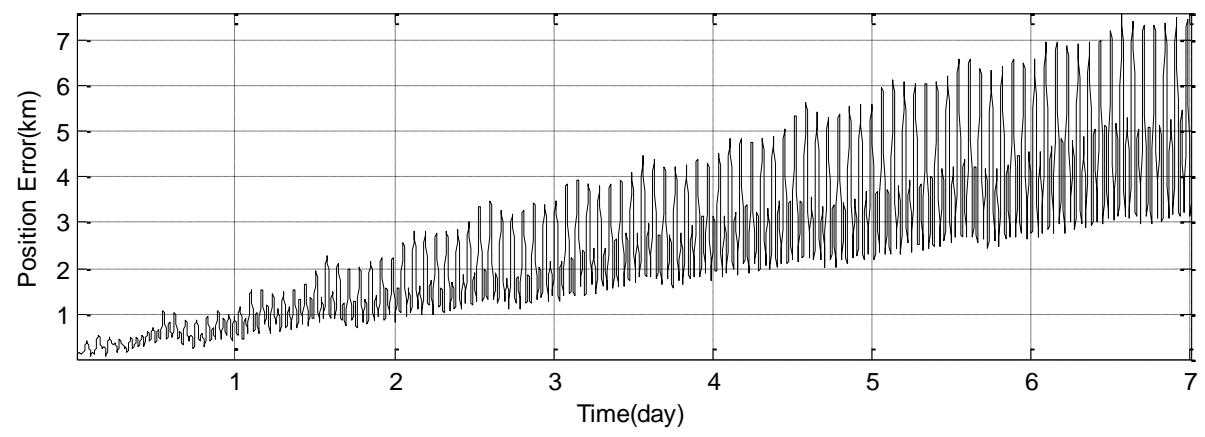

Fig.1 Variations of errors from prediction within 7 days of Larets Satellite

As it can be seen in Fig.1, the position error of the Larets satellite with the height of $691 \mathrm{~km}$ is about $7 \mathrm{~km}$ for seven days prediction. The predition error increases in screw type over time. In the forecast period of seven days, the error fluctuation range is bigger, and the frequency is fast. 


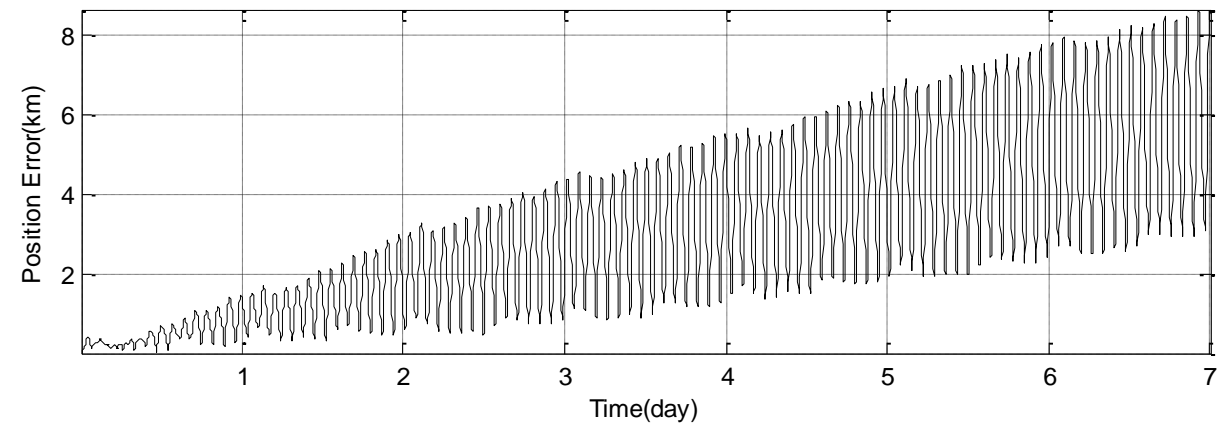

Fig.2 Variations of errors from prediction within 7 days of Ers2 Satellite

As it can be seen in Fig.2, the position error of the Ers 2 satellite with the height of $800 \mathrm{~km}$ is about $8 \mathrm{~km}$ for seven days prediction. The predition error increases in screw type over time as well. But the frequency of the change of the error seem to be slower than the Larets satellite.

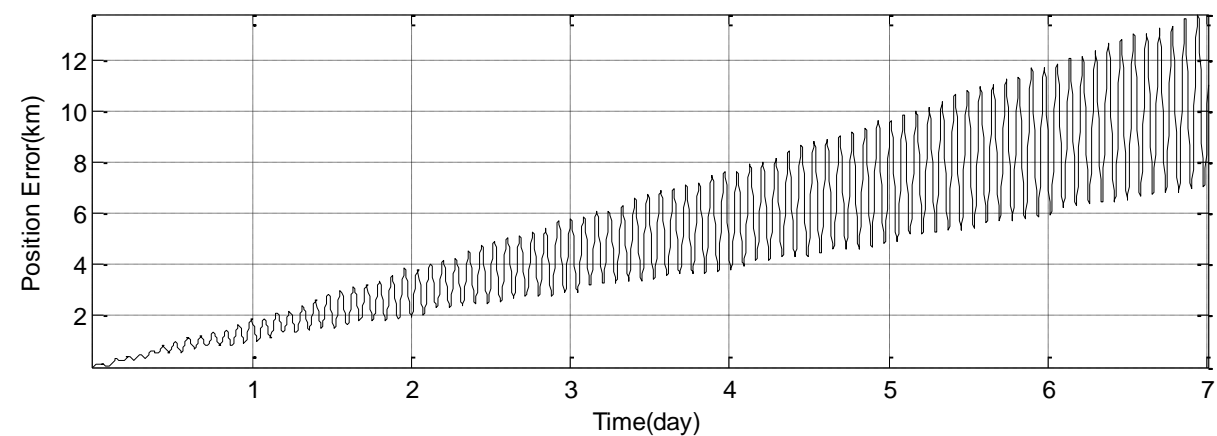

Fig.3 Variations of errors from prediction within 7 days of Ajisai Satellite

As it can be seen in Fig.3, the position error of the Ajisai satellite with the height of $1485 \mathrm{~km}$ is about $14 \mathrm{~km}$ for seven days prediction. The variation of the error is similar with the front two satellites.

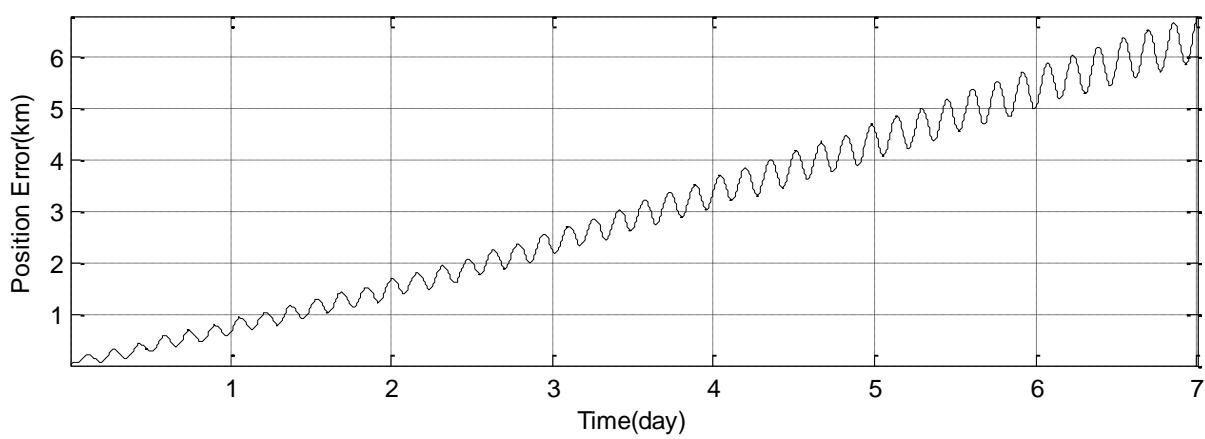

Fig.4 Variations of errors from prediction within 7 days of Lageos1 Satellite

As it can be seen in Fig.4, the position error of the Lageos 1 satellite with the height of $5850 \mathrm{~km}$ is about $7 \mathrm{~km}$ for seven days prediction. The predition error increases in screw type over time just as the three satellites before. The variation range of the error curve is obviously reduced, and the frequency is more slowly.

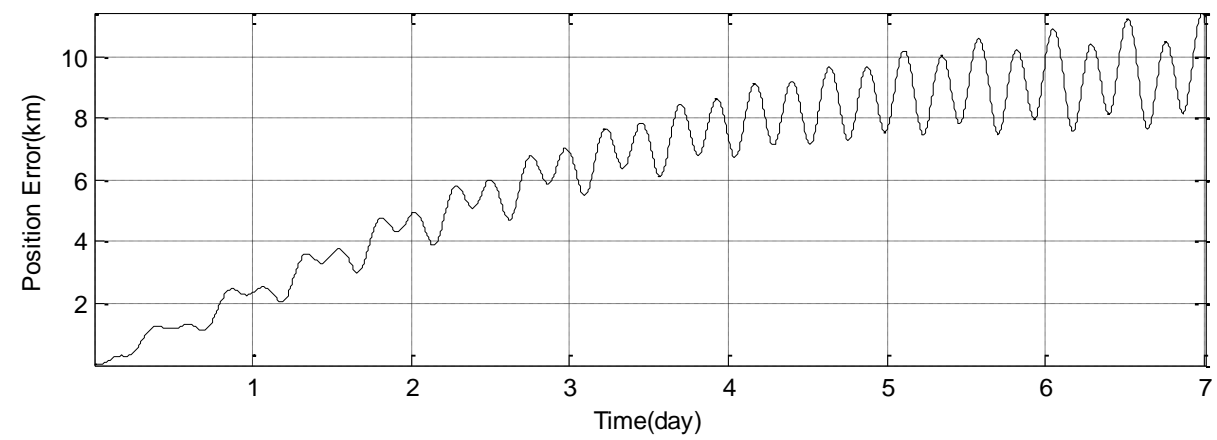

Fig.5 Variations of errors from prediction within 7 days of Etalon1 Satellite 
As it can be seen in Fig.5, the position error of the Etalon1 satellite with the height of $19105 \mathrm{~km}$ is about $12 \mathrm{~km}$ for seven days prediction. The predition error increases in screw type over time. There is no obvious periodicity in the first few days of the curve and it has appeared in the later days. The change range is slightly larger than the Lageos 1 satellite, smaller than the other satellites.

The prediction error curve of the 5 satellites is analyzed, and the following conclusions are drawn:

1) The position errors of the 5 satellites orbit prediction of the MEO and LEO orbit are approximately in $10 \mathrm{~km}$ by using the analysis method.

2) The error increases with time. The lower the height of the satellite is, the faster the oscillation frequency is and the greater the fluctuation range is. The reason may be that the variation of the forecast error is related to the arc length of the satellite motion. The LEO satellite' period is short. In the same forecast period, the number of operation cycles is more and the error changes more quickly.

3) Compared with the SGP4/SDP4 in literatures [1-2], the analysis model for long-term prediction error growth faster in this paper. If we want to further improve the accuracy of prediction, more complex and higher order model should be used.

\section{Summary}

To sum up, this paper briefly introduces an analytical model based on the quasi-analytical average method using the first class of non-singular point root as the basic variable. And then the precision of the model for orbit prediction is analyzed during a short-term of 1day and a long-term of 7 days. The CPF format high precision ephemeris provided by ILRS is used a reference. Numerical results of the simulation demonstrate this model has a good accuracy about several hundred meters in the short term ( 1 day) prediction. The accuracy of the medium and long term ( 7 days) forecast is about $10 \mathrm{~km}$. It can be seen that the model can replace the SGP4/SDP4 model in the short term for the orbit determination of medium and low orbit satellites, and does not depend on the data of TLE. If the parameters such as area mass ratio can be estimated during the precise orbit determination, the precision of the ephemeris will be further improved. A lot of perturbation items are considered in the analytical model. What are the influences of the perturbation term on the accuracy of the orbit prediction and how it happens? All of these problems should be solved in future research to make it perfect.

\section{References}

[1]. Han Lei, Chen Lei, Zhou Bozhao. Precision Analysis of SGP4/SDP4 Implemented in Space Debris Orbit Prediction [J]. Chinese Space Science and Technology, 2004(4): 65.

[2]. Wei Dong, Zhao Changyin. Analysis on the Accuracy of the SGP4/SDP4 Model [J]. Acta Astronomical Sinica, 2009, 50 (3): 333-339.

[3]. Montenbruck O, Eberhard G. Satellite orbits: models, methods and applications [M]. New York: Springer, 2000

[4]. Liu Lin, Hu Songjie, Cao Jianfeng, Tang Jinshi. Theory and Application of spacecraft's Orbit Determination. Beijing: Publishing House of Electronics Industry, 2015.

[5]. Information on: ilrs.gsfc.nasa.gov 